\title{
Call-level Analysis of Hybrid WDM-OCDMA Passive Optical Networks with Finite Traffic Sources
}

\author{
J.S. Vardakas, V.G. Vassilakis, and M.D. Logothetis \\ Wire Communications Laboratory, Dept. of Electrical and Computer Engineering, \\ University of Patras, 265 04, Patras, Greece \\ Email: \{jvardakas, vasilak, m-logo\}@wcl.ee.upatras.gr
}

\begin{abstract}
Passive Optical Networks (PONs) are becoming a mature concept for the provision of enormous bandwidth to end-users with low cost. In this paper we study the call-level performance of two PON configurations: the OCDMA-PON and the Hybrid WDM-OCDMA PON. We propose analytical models for calculating connection failure probabilities (due to unavailability of a wavelength) and call blocking probabilities (due to the total interference on a call that may exceed a permissible threshold) in the upstream direction. The PONs are described/modeled by onedimensional Markov chains. By solving them, we derive recurrent formulas for the blocking probabilities. The proposed analytical models are validated through simulation; their accuracy was found to be absolutely satisfactory.
\end{abstract}

Keywords: Optical code division multiple access, wavelength division multiplexing, passive optical networks, blocking probability, Markov chains, Poisson, quasi-random process

\section{INTRODUCTION}

Optical communications have been envisioned for delivering high-speed services to residential users for over 25 years, but only recently experience intensive growth in the local loop, thanks to Passive Optical Networks (PONs). PONs are the ultimate solution for resolving the last mile bottleneck between high-speed metropolitan networks and the end-users premises. The PON technology has gained increased attention, mainly due to its important advantages, such as low operational and administrational cost, absence of active components between the central office and the customer's premises, uncomplicated upgrade for supporting new services, and provision of huge bandwidth [1], [2].

Current PON configurations are based on the costeffective Time Division Multiplexing (TDM) technology. TDM-based PONs include the Asynchronous Transfer Mode PON (APON) and Broadband PON (BPON) which have already been standardized by the International Telecommunications Union - Telecommunication Standardization Sector (ITU-T) (G.983), as well as the Gigabit PON (GPON; ITU-T G.984) and the Ethernet PON (EPON; IEEE 802.3ah) [3]. Although these TDM-based PON configurations are currently the most popular configurations for providing Fiber-To-The-Home (FTTH) services, a number of next-generation PON architectures have been emerged: a) Wavelength Division Multiplexing (WDM) PONs and b) Optical Code Division Multiple Access (OCDMA) PONs.

The implementation of WDM in PONs is an effective approach for satisfying the future high bandwidth demands coming from the steadily increasing number of users and from bandwidth intensive applications [4]. WDM PONs are usually based on static wavelength allocation, that is, a certain wavelength is dedicated to each Optical Network Unit (ONU) for the upstream and/or the downstream direction. Since the installation of new ONUs requires additional wavelengths, a practical solution is the 
implementation of the Dynamic Wavelength Allocation (DWA) [5]. By using the DWA, the installation of an additional ONU is simplified and the PON can support a high number of ONUs, even more than the number of the wavelengths in the PON.

The successful application of CDMA in wireless systems has challenged the exploitation of its application in the optical communications systems. Recent advantages in device technologies for the optical en/de-coding have renewed the attention on OCDMA [6]. The OCDMA technology holds promise for enhanced security against unauthorized access, fair division of bandwidth and flexibility of the supported bit rate [7].

A call-level analysis of OCDMA systems was discussed in [8], where the teletraffic capacity of an OCDMA network was determined for two Call Admission Control (CAC) schemes. The calculation of the teletraffic capacity in [8] has the restriction of unit call capacity requirement (i.e. single serviceclass), while the presence of the noise distribution is neglected. In this paper, we develop analytical models for the calculation of blocking probabilities in the upstream direction of OCDMA PON (Fig. 1). We calculate Call Blocking Probabilities (CBP), which occur when the total interference on a call exceeds a predefined maximum level. A call is accepted in the upstream direction as long as there are enough resources in the PON. After call acceptance, the signal-to-noise ratio of all in-service calls deteriorates. Because of this, OCDMA systems have no hard limits on call capacity (i.e. the maximum number of calls that the system can support); the fact that a call may be blocked in any system state is expressed by the local blocking probability (LBP). According to the principle of the CDMA technology, a call should be blocked if it increases the noise of all in-service calls above a predefined level, given that a call is noise for all other calls. We consider that calls belong to different service-classes with finite traffic source population and, therefore, we show the applicability of the Engset Multirate Loss Model (EnMLM) [9], on
OCDMA systems. The EnMLM has been proposed for the wired environment of connection-oriented networks in the case of quasi-random call arrival processes [10]. Herein, we extend the EnMLM to incorporate the peculiarities of the OCDMA systems by capturing the LBP and user activity; the latter describes the user behavior by an ON-OFF model. We name the new model, OCDMA-EnMLM (OEnMLM).

Afterwards, the O-EnMLM is extended to cover the case of a hybrid WDM-OCDMA under the DWA scheme, where each ONU has the ability of connecting to the Optical Line Terminal (OLT) by using any available wavelength. In the case that the OLT cannot allocate a free wavelength connection failure occurs. Our study includes the calculation of the Connection Failure Probability (CFP). We also determine the CBP, due to the limited bandwidth capacity of the wavelength, as well as the Total CBP (TCBP) that occurs either due to the inexistence of a free wavelength, or due to the limited bandwidth capacity of the wavelength. All the proposed models are computationally efficient, because they are based on recursive formulas. Our analysis is validated through simulation; the accuracy of the proposed models was found to be quite satisfactory.

The rest of this paper is organized as follows. Section II describes the principles of OCDMA PON modeling; after having presented the multiplexing of OCDMA systems, we first provide the model description in Section A, while in Sections B we determine the LBP. In Section III we propose the OEnMLM. In Section IV we extend the O-EnMLM in order to calculate CFP, CBP and TCBP for the hybrid WDM-OCDMA PON, under the implementation of DWA. Section V is the evaluation Section. We conclude in Section Error! Reference source not found.

\section{PRINCIPLES OF OCDMA MODELING}

In OCDMA, the multiplexing is accomplished by encoding each user's data bit with a unique codeword, 
which is the user's identifier [11]. The encoding procedure is followed by the modulation of a carrier and the transmission of the signal in the optical fiber. After the reception of the signal, along with signals from all other users, the decoding is performed based on the knowledge of the codeword of the desired signal. All other codewords that are not matched at the receiver are spread in order to create a crosscorrelation noise, which is called Multiple Access Interference (MAI). Apart from MAI, other restriction factors on the performance of OCDMA networks are the shot noise, the thermal noise at the receiver and the fiber link noise [12]. It should be noticed that the dominant source of noise is MAI; therefore the cancellation and suspension of MAI is an important problem in OCDMA systems.

\section{A. System Model}

We consider the OCDMA PON of Fig.1, with $N$ ONUs. All ONUs are connected to the OLT through a Passive Optical Combiner (POC). We study the upstream traffic flow direction (from the ONUs to the OLT). At the OLT, a call is not blocked due to the lack of a decoder because we assume a sufficiently large number of optical decoders. Each ONU accommodates $K$ service-classes, while $M_{k}$ is the number of sources that generate calls of service-class $k(k=1, \ldots, K)$; the total number of traffic sources per service-class $k$ in the PON is $N M_{k}$. Due to the fact that calls are generated from a finite number of sources, the call arrival process is quasi-random, where the mean arrival rate of service-class $k$ call per idle source is $\lambda_{k}$ [13]. As far as the service time is concerned, it is exponentially distributed with mean $\mu_{k}^{-1}$. We also consider that each service-class $k$ is characterized by the transmission rate $R_{k}$ (bandwidth per call), the Bit Error Rate (BER) parameter $\left(E b / N_{0}\right)_{k}$ and the user activity factor $v_{k}[14]$.

Because of the OCDMA technology, we need to consider interferences between calls. We distinguish the MAI, $I_{M A I}$, from the shot noise, the thermal noise and the fiber link noise [15]. The latter has a power of
$P_{f}$. The thermal noise is generally modeled as Gauss distribution $\left(0, \sigma_{t h}\right)$, while the shot noise is modeled as a Poisson process where its expectation (mean value) and variance are both denoted by $p$. According to the central limit theorem, we can assume that the additive shot noise is modeled as Gauss distribution $\left(\mu_{s h}, \sigma_{s h}\right)$, considering that the number of users in the PON is relatively large $(M N \geq 10)$. Therefore, the interference $I_{N}$ caused by the thermal noise and the shot noise is modeled as a Gaussian distribution with mean $\mu_{N}=\mu_{s h}$ and variance $\sigma_{N}=\sqrt{\sigma_{t h}^{2}+\sigma_{s h}^{2}}$.

The CAC in the OCDMA-PON system under consideration is performed based on the Noise Rise $(N R)$ measurement, which is defined as the ratio of the total received power at the OLT to the fiber link noise $P_{f}$ :

$$
N R=\frac{I_{M A I}+I_{N}+P_{f}}{P_{f}}
$$

When a new call arrives, the CAC estimates the noise rise and if it exceeds a maximum value, $N R_{\max }$, the new call is blocked and lost. A transformation of (1) yields to the definition of the system load $n$, which is the ratio of the received power from all active users and from the interference $I_{N}$ to the total received power:

$$
n=\frac{I_{M A I}+I_{N}}{I_{M A I}+I_{N}+P_{f}}
$$

The maximum value of the system load $n_{\max }$ corresponds to the maximum value of the noise rise $N R_{\max }$. Similarly to the analysis of the WCDMA wireless system of [14], the load factor $L_{k}$ can be seen as the bandwidth requirement of a service class $k$ call:

$$
L_{k}=\frac{\left(E_{b} / N_{0}\right)_{k} \cdot R_{k}}{W+\left(E_{b} / N_{0}\right)_{k} \cdot R_{k}}
$$

where $W$ is the chip rate of the OCDMA-PON.

The system load can be written as the sum of the load $n_{\text {own }}$ that derives from the active users of the PON and the equivalent load, $n_{N}$ that derives from the 
presence of the shot noise and thermal noise. They are defined in (4) and (5), respectively:

$$
n_{\text {own }}=\sum_{k=1}^{K} m_{k} L_{k}
$$

where $m_{k}$ is the number of active users of serviceclass $k$.

$$
n_{N}=\left(1-n_{\max }\right) \frac{I_{N}}{P_{f}}
$$

According to the adopted CAC policy, the OLT decides whether to accept a new service-class $k$ call or not, by checking the condition:

$$
\beta_{\kappa}\left(n_{N}\right)=P_{k}\left(n_{o w n}+n_{N}+L_{k}>n_{\max }\right)
$$

\section{B. Local Blocking Probability}

Eq. (6) calculates the probability that a new serviceclass $k$ call is blocked, when arriving at any instant, and is called LBP. To calculate it, we use (4) and (5), where the only unknown parameter is the interference caused by the shot noise and the thermal noise, $I_{N}$. As previously mentioned, $I_{N}$ is modeled by a Gaussian distribution $\left(\mu_{N}, \sigma_{N}\right)$. Consequently, because of (5), the load $n_{N}$ that derives from the presence of the shot noise and thermal noise can be modeled by a Gaussian distribution, with mean and variance which are respectively given by:

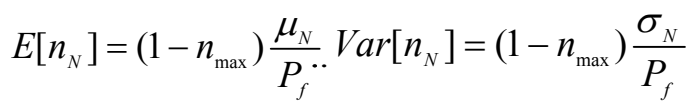

Note that (6) can be rewritten as:

$$
1-\beta_{\kappa}\left(n_{N}\right)=P_{k}\left(n_{N} \leq n_{\max }-n_{\text {own }}-L_{k}\right)
$$

The right-hand side of (8) is the Cumulative Distribution Function (CDF) of $n_{N}$. It is denoted by $F_{n}(x)=P\left(n_{N} \leq x\right)$ and is given by:

$$
F_{n}(x)=\frac{1}{2}\left(1+\operatorname{erf}\left(\frac{\ln (x)-E\left[n_{N}\right]}{\operatorname{Var}\left[n_{N}\right] \sqrt{2}}\right)\right)
$$

where $\operatorname{erf}(\bullet)$ is the well-known error function. Using (8) and (9) we can calculate the LBP, $\beta_{n}$, by means of the substitution $x=n_{\max }-n_{\text {ovn }}-L_{k}$ :

$$
\beta_{n}(x)= \begin{cases}1-F_{n}(x), & x \geq 0 \\ 1, & x<0\end{cases}
$$

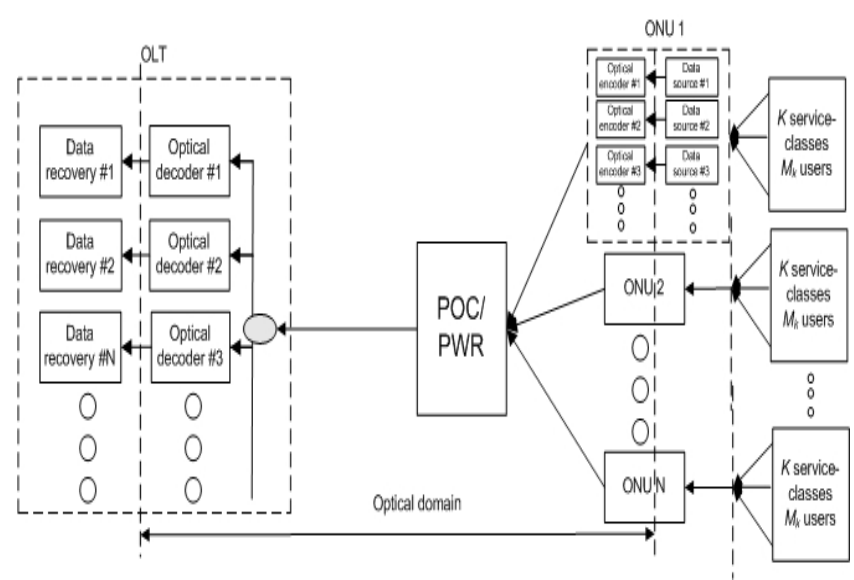

Figure 1: General Configuration of a Passive Optical Network.

\section{THE PROPOSED O-ENMLM}

In order to calculate the occupancy distribution of the bandwidth in the PON, we adopt the Engset Multirate Loss Model (EnMLM) [9]. The system load $n$ is considered as the shared bandwidth capacity of the wavelength and the load factor, $L_{k}$, as the bandwidth requirement of a service-class $k$ call. Since the EnMLM is a discrete state space model, we use a basic load unit, $g$, for the discretization of the system load, $n$ and the load factor, $L_{k}$, in order to derive the system capacity $T$ and the service-class $k$ bandwidth requirement, $b_{k}$ :

$$
T=\frac{n_{\max }}{g} \text { and } b_{k}=\text { round }\left(\frac{L_{k}}{g}\right)
$$

Note that $T$ and $b_{k}$ are measured in bandwidth units (b.u.). Although both active and passive users are present in each ONU, passive users do not consume system bandwidth. A state $i$ in the EnMLM for an OCDMA system, does not represent the total number of occupied b.u., as it happens in the infinite trafficsource model (Erlang Multirate Loss Model-EMLM [16]), but instead, it represents the total number of occupied b.u. when all users are active. The total number of occupied b.u. is $c$. Note that in the EnMLM for an OCDMA system, we have $0 \leq c \leq i$, while in 
EnMLM $c$ is always equal to $i$. When $c=i$, all users are active, while when $c=0$, all users are passive.

Let $q(i)$ be the probability that the system is in state $i$. The bandwidth occupancy $\Lambda(c \mid i)$ is defined as the conditional probability that $c$ b.u. are occupied, when the state is $i$ and is given by [14]:

$$
\Lambda(c i)=\sum_{k=1}^{K} P_{k}(i)\left[v_{k} \Lambda\left(c-b_{k} \mid i-b_{k}\right)+\left(1-v_{k}\right) \Lambda\left(c \mid i-b_{k}\right)\right]
$$

$$
\text { for } i=1, \ldots, i_{\max } \text { and } c \leq i \text {, }
$$

where $\quad \Lambda(0 \mid 0)=1, \Lambda(c \mid i)=0 \quad$ for $c>i \quad$ and $\quad i_{\max }$ represents the highest reachable system state.

In an OCDMA system, due to the presence of MAI, a service-class $k$ call may be blocked at any system state $i$ with probability $L B_{k}(i)$, which is called Local Blocking Factor (LBF):

$$
L B_{k}(i)=\sum_{c=0}^{i} \beta_{k}(c) \Lambda(c \mid i)
$$

The probability $P_{t}(i)$ that state $i$ is reached by a new call of service-class $k$ is given by:

$$
P_{t}(i)=\frac{\left(N M_{k}-n_{k}(i)+1\right) q\left(i-b_{k}\right) \cdot a_{k} \cdot\left(1-L B_{k}\left(i-b_{k}\right)\right) b_{k}}{i \cdot q(i)}
$$

where $N M_{k}$ is the total number of service-class $k$ traffic sources in the PON and $a_{k}=\lambda_{k} / \mu_{k}$ is the offered traffic load per idle traffic source. The probabilities $q(i)$ represent the distribution of the occupied b.u. in the wavelength and can be calculated by extending EnMLM, due to the presence of the local blockings:

$$
i q(i)=\sum_{k=1}^{K} a_{k} \cdot b_{k} \cdot\left(1-L B_{k}\left(i-b_{k}\right)\left(N M_{k}-n_{k}(i)+1\right) q\left(i-b_{k}\right)\right.
$$

for $i>0, q(i)=0$ for $i<0$ and $\sum_{i=0}^{i_{\max }} q(i)=1$.

In order to calculate the distribution $q(i)$ through (15), we need the exact number of service-class calls $n_{k}(i)$, in different system states $i$. This number can be approximated by the average number of service-class $k$ calls with requirement $b_{k}$, when i b.u. are occupied in the system, from these service-classes with infinite number of sources (Poisson arrivals):

$$
n_{k}(i) \approx \frac{N M_{k} \cdot a_{\mathrm{inf}, k} \cdot q_{\mathrm{inf}}\left(i-b_{k}\right) \cdot\left(1-L B_{\mathrm{inf}, k}\left(i-b_{k}\right)\right)}{q(i)}
$$

where $a_{\mathrm{inf}, k}, q_{\text {inf }}$ and $L B_{\text {inf } f, k}$ are the parameters of the corresponding infinite model (EMLM).

The CBP of service-class $k$ are given by can be calculated by adding all the state probabilities multiplied by the corresponding LBFs for all possible system states:

$$
B_{k}=\sum_{i=0}^{i_{\max }} q(i) L B_{k}(i)
$$

The O-EnMLM actually coincides with the Wireless-EnMLM (W-EnMLM) [17], which has been proposed for the call-level analysis of the WCDMA cellular networks. The two models differ in the following points: In the W-EnMLM, we have to consider the so-called inter-cell interference, while in O-EnMLM, no interference from other optical fibers is possible. In the W-EnMLM, only thermal noise is considered as background noise, while in O-EnMLM, we need to consider not only thermal noise but also shot noise and fiber link noise. Moreover, in the case of both the thermal and shot noise we have taken into account the distributions of these noise sources (not only the average noise power).

\section{BLOCKING ANALYSIS IN HYBRID WDM-OCDMA PON}

We consider the hybrid TDM-WDM PON of Fig.1, with $N$ ONUs, where the POC is replaced by a Passive Wavelength Router (PWR). The PWR is a passive combiner/splitter device, which is responsible for the routing of multiple wavelengths in a single fiber toward the OLT [18]. The PON supports $C$ wavelengths and $K$ service-classes (same serviceclasses per ONU). Calls of service-class $k$ arrive to the ONU from a finite number of sources $M_{k}$ and are groomed onto one wavelength. Each service-class $k$ call requires $b_{k}$ b.u. from the wavelength, in order to be serviced (fixed bandwidth requirement). The call arrival process is quasi-random where the mean arrival rate of service-class $k$ per idle source is $\lambda_{k}$. The 
call-service time is exponentially distributed with mean $\mu_{k}^{-1}$.

The connection establishment procedure between an ONU and the OLT is based on the DWA procedure. When a call arrives at an ONU, while no other calls of this ONU are in service, it requests for an available wavelength from the OLT. If a free wavelength is found by the OLT, it is assigned to the ONU to establish the connection (and service the call). If more than one free wavelength is available, one of these wavelengths is selected randomly. If a free wavelength cannot be found, connection failure occurs and the call is considered blocked and lost. After the connection establishment, all calls from the same ONU are serviced through the same wavelength, as long as they can be accepted according to the CAC procedure in the wavelength (otherwise, the calls are blocked and lost). When all calls on a wavelength terminate the connection also terminates and the wavelength becomes available to any new arriving call from any ONU.

\section{A. Connection Failure Probability}

The calculation of CFP is based on the knowledge of the occupancy distribution of the wavelengths in the PON. To this end, we formulate a Markov chain with the state transition diagram of Fig. 2, where the state $j$ represents the number of occupied wavelengths in the PON. We denote the total arrival rate of calls from an ONU by $\lambda=\sum_{k=1}^{K} M_{k} \lambda_{k}$, since all traffic sources of the ONU are idle, before the connection establishment. A connection establishment (from any ONU) is realized with a rate that depends on the number of ONUs, which have not established a connection yet, and the number of the occupied wavelengths. Thus, the transition from state $[j-1]$ to state $[j]$ of the Markov chain occurs $[N-(j-1)] \lambda$ times per unit time, because in state $[j-1]$ the number of the ONUs which have not established a connection is $N$ $(j-1)$, while the call arrival rate is aggregated to $\lambda$, since a call from any service-class is required for the connection establishment. The reverse transition, from state $[j]$ to state $[j-1]$ is realized $j Q$ times per unit time, where $Q$ is the service rate of a wavelength. The rate $Q$ can be determined by the product of (the conditional probability that $b_{k}$ b.u. are occupied in the wavelength by only one call of service-class $k$, given that the wavelength is occupied) by (the corresponding service rate $\mu_{k}$ ).

$$
Q=\sum_{k=1}^{K} \mu_{k} y_{k}\left(b_{k}\right) \hat{q}\left(b_{k}\right)=\sum_{k=1}^{K} \mu_{k} y_{k}\left(b_{k}\right) \frac{q\left(b_{k}\right)}{\sum_{i=1}^{T} q(i)}
$$

where $y_{k}(i)$ is the mean number of service-class $k$ calls in the wavelength, when $i$ b.u. are occupied in the wavelength, and $q(i)$ is the occupancy distribution of the b.u. inside a wavelength, and $\hat{q}(i)$ is the conditional probability that $i$ b.u. are occupied, given that the wavelength is occupied (at least one call is serviced through the wavelength), according to the EnMLM. In order to calculate the service rate Q we have to consider that the release of a wavelength is realized through the service of its last call which may belong to any service-class.

The release of a wavelength occurs when the number of occupied b.u. in the wavelength is $b_{k}$, since only one service-class $k$ call occupy system resources. Therefore, the release of this last call occurs with a rate $\mu_{\kappa} \cdot y_{k}\left(b_{k}\right) \cdot \hat{q}\left(b_{k}\right)$.

The probability $P(j)$ that $j$ wavelengths are occupied in the link can be derived from the rate balance equations of the state transition diagram of Fig. 2 (a method for deriving the distribution $P(j)$, for $j=0,1$, $\ldots, C$, can be found in [1]):

$$
P(j)=\left(\frac{\lambda}{Q}\right)^{j} \cdot \frac{\prod_{i=1}^{j}(R-i+1)}{j !} \cdot\left[\sum_{i=0}^{C}\left(\frac{\lambda}{Q}\right)^{i} \cdot \frac{\prod_{j=1}^{i}(R-j+1)}{i !}\right]^{-1}
$$

The CFP is determined by $\mathrm{P}(C)$, since a connection establishment is blocked and lost, if and only if all the wavelengths are occupied.

The probability $P(j)$ is valid for any WDM PON configuration, according to the multiple access 
technique that is used in each wavelength (different $\lambda$ and $Q$ result from each technique).

\section{B. Call Blocking Probabilities}

The CBP of service-class $k$ calls of a particular ONU utilizing the access network can be calculated considering that, the blocking states for a service-class $k$ call are the last $b_{k}$ states in the occupancy distribution of a wavelength. Therefore, the CBP is given by (17).

The TCBP is the probability that a call is lost, either due to the restricted bandwidth capacity of a wavelength, or due to the unavailability of a free wavelength in the PON. The calculation of the TCBP is realized by considering the probability that a call is accepted for service. This situation occurs, either when this call is the first call that arrives at an ONU that has not established a connection yet, and a free wavelength can be found for the connection establishment, or this call arrives at an ONU that has already established a connection while, at the same time, enough free b.u. are available in the wavelength. The probability $P_{\text {accept }}^{k}$ that a service-class $k$ call is accepted for service by the PON is given by:

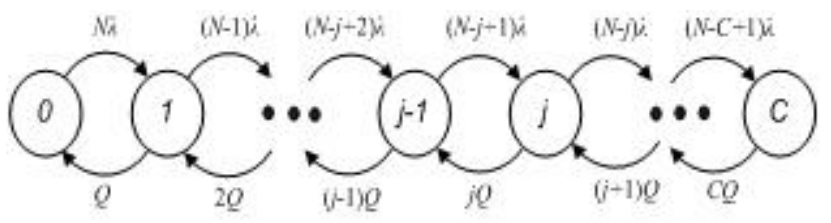

Figure 2: State transition diagram of a WDM-OCDMA PON with $C$ wavelengths and dynamic wavelength assignment.

$$
P_{\text {accept }}^{k}=P_{s} \cdot \frac{\sum_{i=1}^{j_{\max }-b_{k}} q(i)}{\sum_{i=1}^{j_{\max }} q(i)}+\left(1-P_{s}\right) \cdot(1-P(C))
$$

where $P_{s}$ is the probability that an ONU has already established a connection upon the call arrival. The first term of the right-hand side of (20) signifies the probability that a service-class $k$ call has arrived to an ONU, which has already established a connection (with probability $P_{s}$ ) and there are at least $b_{k}$ b.u. available in the wavelength (with probability that is given by the fraction of (20)). The second term of the right-hand side of (20) refers to the probability, that a service-class $k$ call arrives at the ONU, which has not established a connection (probability $1-P_{s}$ ), and there is at least one available wavelength in the PON (probability $1-P(C)$ ). The probability $P_{s}$ is given by the summation of all multiplications of the probabilities $P(j)$ by the probability that a specific ONU has established a connection, for $j=1, \ldots, C$ :

$$
P_{s}=\sum_{j=1}^{C} P(j) \frac{\left(\begin{array}{c}
N-1 \\
j-1
\end{array}\right)}{\left(\begin{array}{c}
N \\
j
\end{array}\right)}=\sum_{j=1}^{C} P(j) \frac{j}{N}
$$

Combining (20) and (21), we can express TCBP of service-class $k$ calls as:

$$
T B_{k}=1-P_{a c c e p t}^{k}
$$

\section{EVALUATION}

In this section we evaluate the accuracy of the presented analytical models through simulation. To this end, we using the SIMSCRIPT II.5 simulation tool, we have simulated the two models that were presented in sections III and IV. The simulation results have been obtained as mean values of 6 runs with confidence interval of $95 \%$. However, the resultant reliability ranges of the simulation results are very small; therefore we present only the mean values. The evaluation of the analytical models is realized by considering two examples. In the first example, we consider an OCDMA-PON with $N=20$ ONUs.

The chip rate of the upstream direction is selected to be 1.5 Gcps. The PON accommodates 2 serviceclasses with transmission rates $R_{1}=24 \mathrm{Mbps}$ and $R_{2}=32 \mathrm{Mbps}$, respectively, while the BER parameters are $\left(E_{b} / N_{0}\right)_{1}=4 \mathrm{~dB}$ and $\left(E_{b} / N_{0}\right)_{2}=3 \mathrm{~dB}$, respectively. The activity factor of the service-class $s_{1}$ is $v_{1}=1$ and of the service-class $s_{2}$ is $v_{2}=0.3$. The number of trafficsources of the service-class $s_{1}$ is $M_{1}=5$ and of the service-class $s_{2}$ is $M_{2}=5$. The interference caused by the presence of the shot noise and the thermal noise is 
modeled as a Gaussian distribution with parameters $\left(\mu_{N}=\sigma_{N}=10^{-15} \mathrm{~mW}\right)$. We assume that the maximum system load $n_{\max }$ is set to 0.9 , while the fiber link noise power is $P_{f}=-180 \mathrm{dBm}$. The analytical results for the CBP are obtained through (1)-(18). We take measurements for 6 different traffic-load points (xaxis of Fig.3). Each traffic load point corresponds to some values of the offered traffic-load of both serviceclasses, as it is shown in Table I. In Fig.3 we present the analytical and simulation results for the CBP, versus the offered traffic-load. As the results reveal, the accuracy of the proposed models is quite satisfactory.

\begin{tabular}{|l|l|l|l|l|}
\hline \multirow{2}{*}{} & \multicolumn{2}{|c|}{$1^{\text {st }}$ example } & \multicolumn{2}{c|}{$2^{\text {nd }}$ example } \\
\cline { 2 - 5 } & $a_{1}$ & $a_{2}$ & $a_{1}$ & $a_{2}$ \\
\hline 1 & 0.02 & 0.04 & 0.01 & 0.02 \\
\hline 2 & 0.04 & 0.08 & 0.02 & 0.04 \\
\hline 3 & 0.06 & 0.12 & 0.03 & 0.06 \\
\hline 4 & 0.08 & 0.16 & 0.04 & 0.08 \\
\hline 5 & 0.10 & 0.20 & 0.05 & 0.10 \\
\hline 6 & 0.12 & 0.24 & 0.06 & 0.12 \\
\hline
\end{tabular}

Table 1: Offered Traffic-Load for the Evaluation examples.

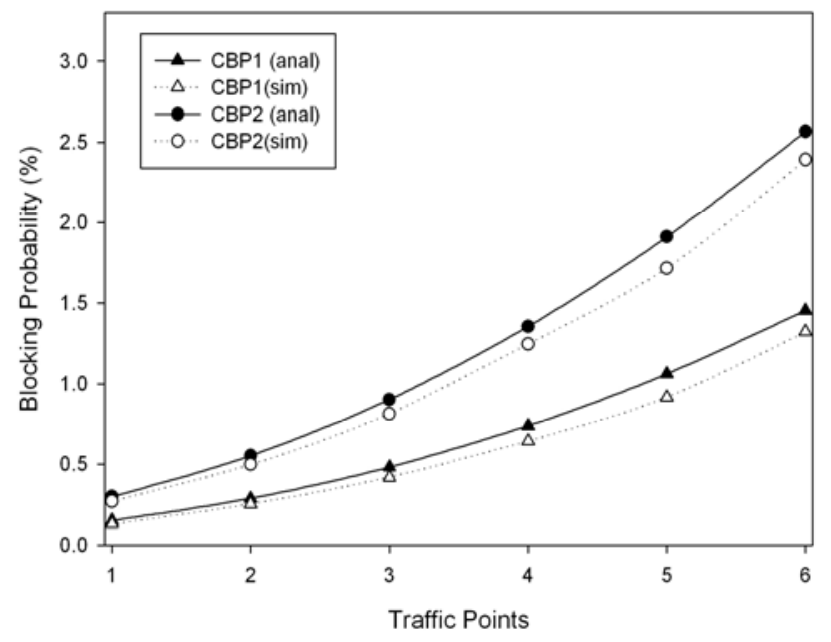

Figure 3: CBP results versus the offered traffic-load for the two service-classes in the $1^{\text {st }}$ example.

The effect of the number of sources in the CBP can be monitored in Fig. 4, where the product $N M_{k} a_{k}$ is kept constant $\left(N M_{1} a_{1}=0.08 \mathrm{erl}\right.$ and $\left.N M_{2} a_{2}=0.16 \mathrm{erl}\right)$. In all cases the model's accuracy is satisfactory. We notice that increasing the number of sources the CBP also increases. It is due to the fact that when we have a larger number of sources, the percentage of idle sources is higher, leading to a higher offered trafficload and therefore to higher values of blocking probabilities.

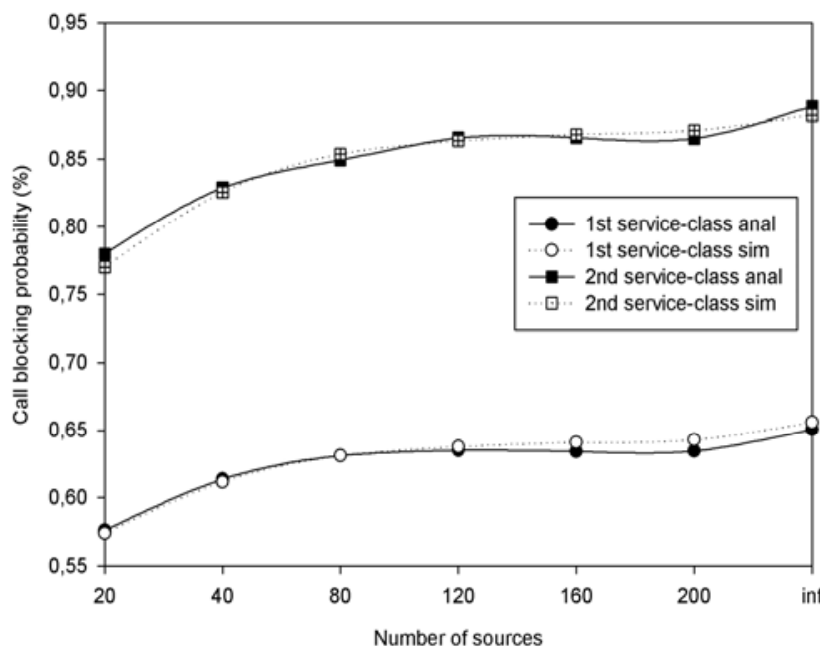

Figure 4: $C B P$ versus the number of traffic sources in the $1^{\text {st }}$ example.

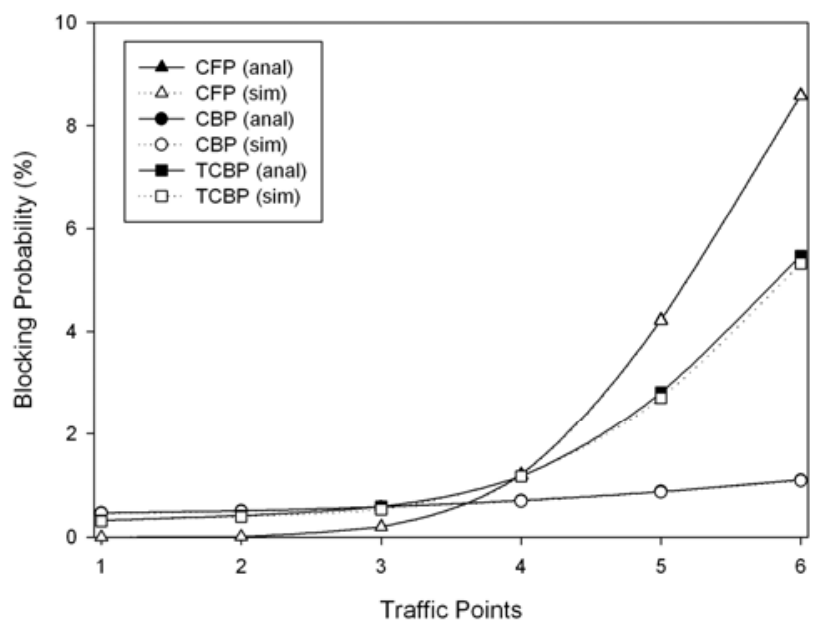

Figure 5: CFP, CBP and TCBP results versus the offered traffic-load for the $1^{\text {st }}$ service-class in the $2^{\text {nd }}$ example.

In the second example we evaluate the analytical model for the hybrid WDM-OCDMA PON case. The PON accommodates $C=32$ wavelengths, while the chip rate of a wavelength in the upstream direction is 1.5 Gcps. We assume that the hybrid WDM-OCDMA PON accommodates the same two service-classes, as 
in the first example. In Fig. 4 and 5 we present analytical and simulation results for the CFP, CBP and TCBP versus the offered traffic-load for the service-classes $s_{1}$ and $s_{2}$, respectively. We consider 6 traffic-load points $(1,2, \ldots, 6)$ in the $\mathrm{x}$-axis of Fig. 4 and 5 , where each point corresponds to some values of the traffic-load of both service-classes, as it is shown in Table 1. The analytical results for the CFP are obtained through (14)-(16) and (18)-(20), the CBP analytical results through (1)-(18), while the TCBP analytical results are obtained through (16) and (20)(22). The comparison of the analytical and the corresponding simulation results of Fig. 4 and 5 show satisfactory accuracy of the proposed model.

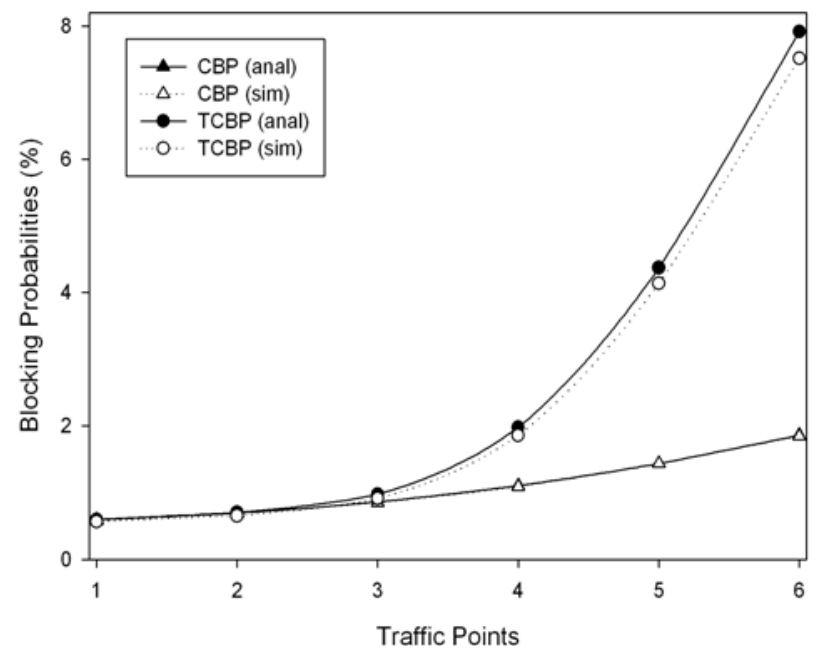

Figure 6: $C B P$ and TCBP results versus the offered traffic-load for the $2^{\text {nd }}$ service-class in the $2^{\text {nd }}$ example

\section{CONCLUSION}

In conclusion, we propose a teletraffic loss model for the call-level analysis of the upstream direction of an OCDMA PON. This model is extended in order to provide an analytical loss model of a hybrid WDMOCDMA PON. Both systems accommodate different service-classes with a finite number of traffic sources. We provide formulas for the calculation of the CFP, CBP and TCBP. The accuracy of the proposed calculations is quite satisfactory as was verified by simulations. In our future work we shall extend this analysis, in order to study the downstream direction (from the OLT to the ONUs) and the impact of different noise distributions, of the attenuation and the dispersion, in the system's performance.

\section{ACKNOWLEDGMENT}

The authors would like to thank Dr Ioannis D. Moscholios (University of Patras, Greece) for his support.

\section{REFERENCES}

[1] L.G Kazovsky, W-T Shaw, D. Gutierrez, N. Cheng and S-W Wong, "Next-Generation Optical Access Networks", Journal of Lightwave Technology (Invited Paper), Vol. 25, No. 11, pp. 3428-3442, November 2007.

[2] P. W. Shumate, "Fiber-to-the-Home: 1977-2007", IEEE/OSA Journal of Lightwave Technology (Invited Paper), Vol. 26, No. 9, pp. 1093-1103, May 2008.

[3] F. Effenberger, D. Cleary, O. Haran, G. Kramer, R. Li, M. Oron, T. Pfeiffer, "An Introduction to PON Technologies", IEEE Communications Magazine, March 2007.

[4] K. Kitayama, X. Wang and N. Wada, "OCDMA over WDM PON -A Solution Path to Gigabit-Symmetric FTTH", Journal of Lightwave Technology. Vol.24, April 2006.

[5] A. R. Dhaini, C. M. Assi, M. Maier, and A. Shami, "Dynamin Wavelength and Bandwidth Allocation in Hybrid TDM?WDM EPON Networks", IEEE/OSA Journal of Lightwave Technology, Vol. 25, No, 1, January 2007, pp.277-286

[6] J. P. Heritage, A. M. Weiner, "Advances in Spectral Optical Code-Division Multiple-Access Communications", IEEE Journal of Selected Topics in Quantum Electronics, Vol. 13, No. 5, September/October 2007.

[7] K. Fouli and M. Maier, "OCDMA and Optical CodingPrinciples, Applications, and Challenges", IEEE Communications Magazine, pp. 27-34, August 2007.

[8] S. Goldberg and P.R. Prucnal, "On the Teletraffic Capacity of Optical CDMA", IEEE Transactions on Communications, Vol. 55, No. 7, July 2007, pp. 13341343.

[9] G. Stamatelos, V. Koukoulidis, Reservation-based bandwidth allocation in a radio ATM network, IEEE/ACM Transactions on Networking, Vol. 5, No. 3, pp. 420-428, 1997. 
[10] I. D. Moscholios, M. D. Logothetis and G. K. Kokkinakis "On the Calculation of Blocking Probabilities in the Multi-Rate State-Dependent Loss Models for Finite Sources", Mediterranean Journal of Computers and Networks, Vol. 3, No. 3, pp. 100-109, July 2007.

[11] A. E. Willner, P. Saghari, and V. R. Arbab, "Advanced Techniques to Increase the Number of Users and Bit Rate in OCDMA Networks", IEEE Journal of Selected Topics in Quantum Electronics, Vol 13, No. 5, September, October 2007, pp.1403-1414.

[12] Z. ChongFu, Q. Kun, and X. Bo, "Passive Optical Networks on Optical CDMA: Design and System Analysis", Chinese Science Bulletin, January 2007, Vol. 52, No. 1, pp. 118-126.

[13] H. Akimaru, K. Kawashima, Teletraffic - Theory and Applications, Springer-Verlag, 1993.

[14] D. Staehle and A. Mäder, “An analytic approximation of the uplink capacity in a UMTS network with heterogeneous traffic," in proc. 18th ITC18, Sept. 2003, Berlin.

[15] W. Ma, C. Zuo, H. Pu, and J. Lin, "Performance Analysis on Phase-Encoded OCDMA Communication System", Journal of Lightwave Technology, Vol. 20, No. 5, May 2002.

[16] I. D. Moscholios, M. D. Logothetis, and G. K. Kokkinakis, "Connection Dependent Threshold Model: A Generalization of the Erlang Multiple Rate Loss Model," Performance Evaluation, Special issue on "Performance Modeling and Analysis of ATM \& IP Networks", Vol.48, issue 1-4, pp. 177-200, May 2002.

[17] V. G. Vassilakis, G. A. Kallos, I.D. Moscholios, and M. D. Logothetis, "The Wireless Engset Multi-Rate Loss Model for the Call-level Analysis of W-CDMA Networks", in proc. of $18^{\text {th }}$ IEEE Personal, Indoor and Mobile Radio Communications Symposium 2007 (PIMRC'07) Athens 2007.

[18] K. Grobe, and J-P. Elbers, "PON in Adolescence: From TDMA to WDM-PON", IEEE Communications Magazine, Vol. 46, No. 1, January 2008, pp. 26-34.

[19] John S. Vardakas, Vassilios G. Vassilakis, and Michael D. Logothetis, "Blocking Analysis in Hybrid TDMWDM PONs Supporting Elastic Traffic", in proc. of the $4^{\text {th }}$ Advanced International Conference on Communications, (AICT 2008), Athens, Greece, 8-13 June 2008.

\section{AUTHOR BIOGRAPHIES}

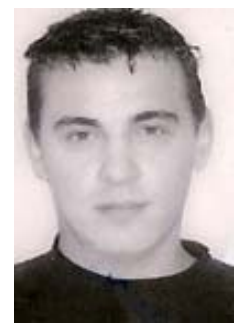

John S. Vardakas was born in Alexandria, Greece, in 1979. He received his Dipl. - Eng. degree in Electrical and Computer Engineering from the Democritus University of Thrace, Greece, in 2004. Since 2005 he is a Ph.D student at the Wire Communications Laboratory, in the Division of Communications and Information Technology of the Department of Electrical and Computer Engineering, University of Patras, Greece. His research interests include teletraffic engineering in optical and wireless networks. He is a member of the Optical Society of America (OSA) and the Technical Chamber of Greece (TEE).

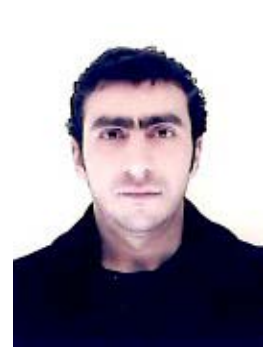

Vassilios G. Vassilakis was born in Sukhumi, Georgia, in 1978. He received a Dipl.-Eng. degree in Computer Engineering \& Informatics from the University of Patras, Greece, in 2003. Since 2004, he is a Ph.D student at the Wire Communications Laboratory, in the Division of Communications and Information Technology of the Department of Electrical and Computer Engineering, University of Patras, Greece. $\mathrm{He}$ is involved in national research and $R \& D$ projects. His main research interest is on teletraffic theory and engineering and QoS assessment in wireless networks. He is a member of the Technical Chamber of Greece (TEE). 


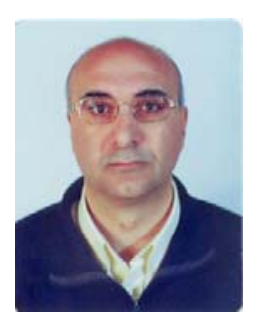

Michael D. Logothetis was born in Stenies, Andros, Greece, in 1959. He received his Dipl.-Eng. degree and Doctorate in Electrical Engineering, both from the University of Patras, Patras, Greece, in 1981 and 1990, respectively. From 1982 to 1990 , he was a Teaching and Research Assistant at the Laboratory of Wire Communications, University of Patras, and participated in many national and EU research programmes, dealing with telecommunication networks, as well as with office automation (including natural language processing). From 1991 to 1992 he was Research Associate in NTT's Telecommunication Networks Laboratories, Tokyo, Japan. Afterwards, he was a Lecturer in the Department of Electrical \& Computer Engineering of the University of Patras; in 2003, he became Associate Professor, and recently (2009) he was elected (Full) Professor, in the same Department. He currently teaches the courses: Broadband Telecom Networks; Telecom Systems I;
Computer Networks, Teletraffic Theory, and Introduction to Communications. His research interests include teletraffic theory and engineering, traffic/network control, simulation and performance optimization of telecommunications networks. He has published over 100 conference/journal papers (more than 20 journals during the last seven years) and has over 200 third-party citations. He has published a teletraffic book in Greek. He has organized the $5^{\text {th }}$ International Conference on Communications Systems, Networks and Digital Signal Processing, CSNDSP 2006. He is serving on the Technical Program Committee of several international conferences. He has organized and chaired several technical sessions and has offered tutorial lectures in conferences. Also, he has become a Guest Editor in several journals. $\mathrm{He}$ is a member of the IARIA (Fellow Member), IEEE (Senior Member), IEICE, ETRI, FITCE and the Technical Chamber of Greece (TEE). 